\title{
Adolescent Plaque Brachytherapy for Large Choroidal Metastasis from Lung Carcinoid Tumor
}

\author{
Marcos A. Crespo ${ }^{a}$ Victor M. Villegas ${ }^{a, b}$ c Maria E. Echevarriad \\ Carmen M. Gurrea ${ }^{e}$ f Timothy G. Murray ${ }^{g}$ Carlos M. Chevere ${ }^{\text {h }}$
}

${ }^{a}$ Department of Surgery, Ponce Health Sciences University, Ponce, PR, USA; ${ }^{b}$ Department of Ophthalmology, University of Puerto Rico, Medical Sciences Campus, San Juan, PR, USA; 'Bascom Palmer Eye Institute, University of Miami Miller School of Medicine, Miami, FL, USA; d Department of Pediatrics, University of Puerto Rico, Medical Sciences Campus, San Juan, PR, USA; ${ }^{e}$ CMG Pathology Lab, Corp, San Juan Health Centre, San Juan, PR, USA; fDepartment of Pathology and Laboratory Medicine, Cardiovascular Center of Puerto Rico and the Caribbean, San Juan, PR, USA; 9Murray Ocular Oncology and Retina (MOOR), Miami, FL, USA; hDepartment of Radiation Oncology, Hospital Oncológico, HIMA San Pablo, Caguas, PR, USA

\section{Keywords}

Brachytherapy · Carcinoid tumor · Choroidal metastasis · Pediatric carcinoid · Plaque radiotherapy

\begin{abstract}
The purpose of this publication is to report a child with choroidal metastasis from a primary lung carcinoid tumor treated with 125-iodine plaque brachytherapy while achieving complete tumor regression. A 15-year-old girl with a history of stage IV lung carcinoid tumor developed painless progressive visual loss in her right eye. Ophthalmological exam documented a unilateral solitary large choroidal mass with complete exudative retinal detachment. Planned palliative treatment with plaque brachytherapy was performed utilizing a total dose of $45 \mathrm{~Gy}$ to the tumor apex. No tumor regression was noted after 6 months of follow-up. Ten months after primary therapy, re-treatment with a total dose of $90 \mathrm{~Gy}$ to the tumor apex was performed due to tumor progression. Complete regression was achieved after secondary treatment without evidence of recurrence after 18 months of follow-up. In conclusion, high-dose plaque brachytherapy may induce complete tumor regression in select adolescent patients with choroidal metastasis from lung carcinoid tumor.
\end{abstract}




\section{Introduction}

Carcinoid tumors are a rare neuroendocrine tumor that arise from enterochromaffin cells. These tumors secrete biogenic amines, peptides, and tachykinins that may lead to the carcinoid syndrome [1]. Carcinoid may arise from various locations. However, the majority develop within the gastrointestinal tract (54.5\%) and pulmonary system (30.1\%). Less frequently, carcinoid tumors may arise from the pancreas, biliary tract, head and neck, and genitourinary system [2]. Unlike other neuroendocrine malignant tumors, mostlung carcinoid tumors are diagnosed early with stage IV metastatic involvement being relatively uncommon [3]. The principal sites of extranodal metastasis for carcinoid tumor are the liver (44.1\%), lung (13.6\%), and pancreas (6.8\%) [4].

Intraocular metastasis from carcinoid tumors is extremely rare [5]. Most reports of uveal metastasis from carcinoid tumors have been associated with primary bronchial tumors [5]. Bronchial carcinoids are rare tumors in the pediatric population with an estimated incidence of 0.6 per million $[6,7]$. The literature regarding choroidal metastasis from carcinoid tumors in the pediatric population is scarce with only 3 previous reports [8-10]. In this case report, we document a child with choroidal metastasis from bronchial carcinoid tumor that was treated with high-dose plaque brachytherapy with complete ocular tumor resolution.

\section{Case Report/Case Presentation}

A 15-year-old female was referred to our clinic due to painless visual loss in her right eye (OD) of 6-month duration. Past medical history included a right lung mass first diagnosed by CT 3 years before. The subject had also undergone right middle and lower lobectomies with endobronchial mass excision. However, lymph node biopsy was not performed despite enlargement on initial chest CT imaging. She was then lost to follow-up after excision due to Hurricane Maria.

Ophthalmologic examination was remarkable for a solitary nasal amelanotic orange-colored choroidal mass with associated exudative retinal detachment OD (Fig. 1A). The contralateral eye was unremarkable. Ultrasonography showed a nasal choroidal mass OD measuring 14.4 $\times 11.7 \times 7.8 \mathrm{~mm}$ (Fig. 1B).

Histopathologic review of the prior endobronchial specimen was consistent with a typical bronchial carcinoid ( $<2$ mitoses per $2 \mathrm{~mm}^{2}$ and no necrosis) showing both organoid and trabecular patterns (Fig. 1C, D). The excised lung tumor measured $3.5 \mathrm{~cm}$ in its greatest dimension and did not invade adjacent structures. The carcinoid tumor was low grade and well differentiated with Ki-67 3\%. Immunohistochemical stains where positive for NSE, CHRA, SYN, and CD56 and focally positive for CK AE1/AE3.

CT scans of the orbit, chest, abdomen, and pelvis were performed for staging and showed solid lesions in the liver and lung, enlarged mediastinal lymph nodes, and sclerotic lesions in the thoracolumbar spine and pelvic bones, all of which are consistent with stage IV disease. In addition, nuclear medicine bone scan showed lesions suggestive of metastatic disease around both orbits, multiple ribs, L3 vertebral body, and pelvic bones (Fig. 2A, B).

Systemic chemotherapy was started, but no regression has been observed. Multiple agents have been used without success (Table 1). Orbital MRI was consistent with a right eye choroidal mass. Brain MRI did not show any intracranial lesions. Octreotide scan showed findings consistent with previous radiologic studies (Fig. 2C, D). Urinalysis was negative for 5-hydroxyindoleacetic acid and chromogranin A.

The subject underwent palliative plaque brachytherapy with a total dose of 45 Gy to the tumor apex. Brachytherapy localization was confirmed with sonography at the time of surgery. Tumor regression was not present after therapy. No local adjuvant therapies were undertaken

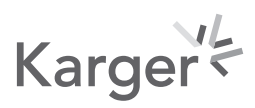




\section{Case Reports in Oncology}

\begin{tabular}{l|l}
\hline Case Rep Oncol 2021;14:1483-1489 \\
\hline DOI: 10.1159/000519045 & $\begin{array}{l}\text { @ 2021 The Author(s). Published by S. Karger AG, Basel } \\
\text { www.karger.com/cro }\end{array}$ \\
\hline
\end{tabular}

Crespo et al.: Adolescent Plaque Brachytherapy for Choroidal Metastasis from Carcinoid Tumor
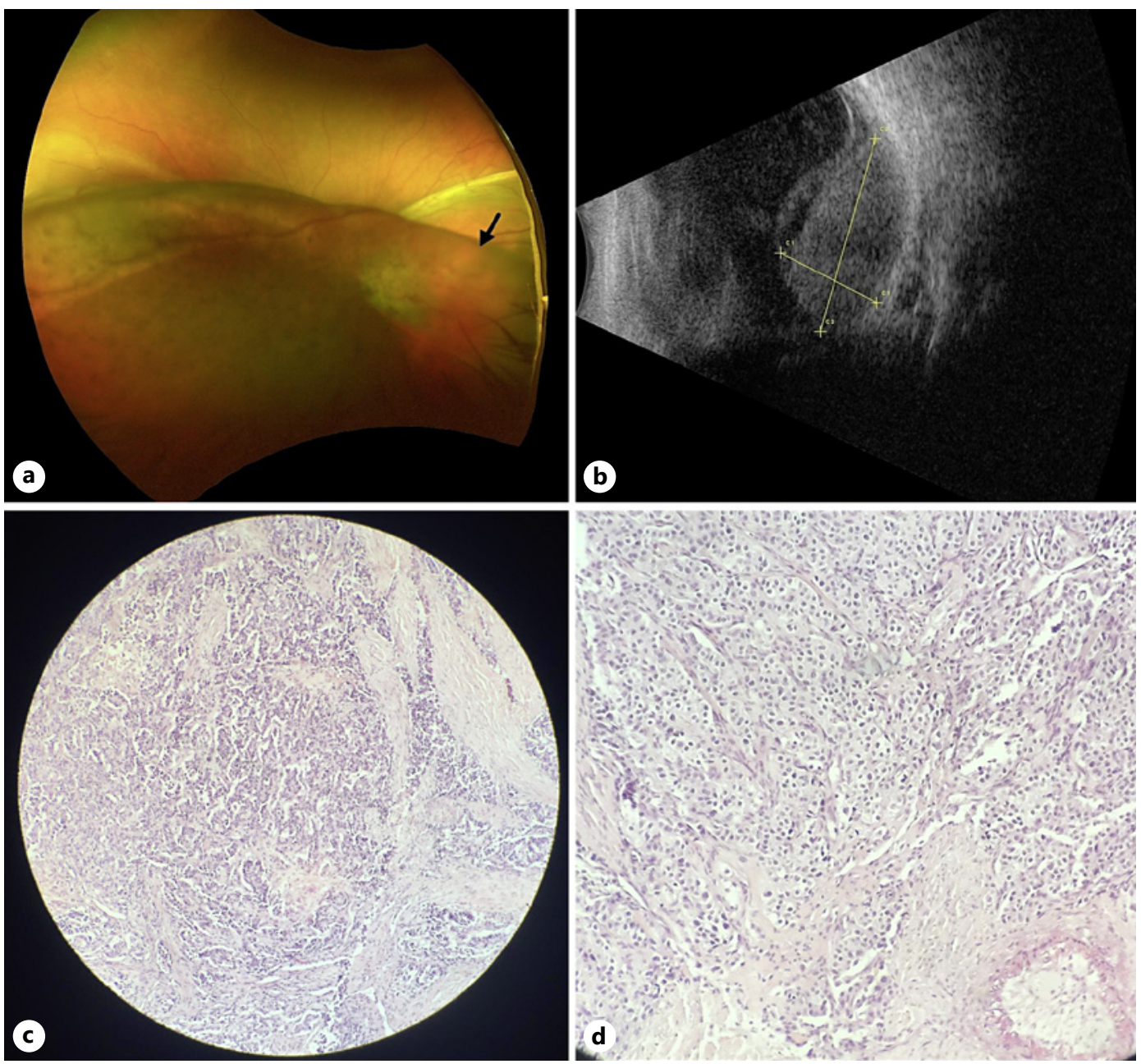

Fig. 1. a Fundus photograph of an inferonasal choroidal mass (arrow) with associated serous retinal detachment. b B-scan of the right eye shows a hyperechoic choroidal mass with an apical height of $7.8 \mathrm{~mm}$ and associated retinal detachment. c Low-magnification histopathological analysis of bronchial carcinoid tumor showing trabecular pattern. d High magnification shows organoid nesting arrangement with fine vascular stroma, moderate amount of eosinophilic cytoplasm, and finely granular nuclear chromatin.

after brachytherapy. Ten months after primary treatment, tumor progression was seen in association with neovascular glaucoma (Fig. 3A).

Re-treatment with plaque brachytherapy was performed with a total dose of $90 \mathrm{~Gy}$ (second fraction of $45 \mathrm{~Gy}$ ) to the tumor apex. One month after radiotherapy, significant tumor regression was present (Fig. 3B). Eighteen months after secondary treatment, the patient continues to be stable without any evidence of local intraocular recurrence despite systemic progression of her disease (Fig. 3C).

\section{Discussion}

Uveal metastasis is the most common intraocular malignancy in adults [11]. However, choroidal metastasis from carcinoid tumor is exceedingly rare, and even more so, in the pediatric population [8-10]. Previously described clinical characteristics associated with choroidal 

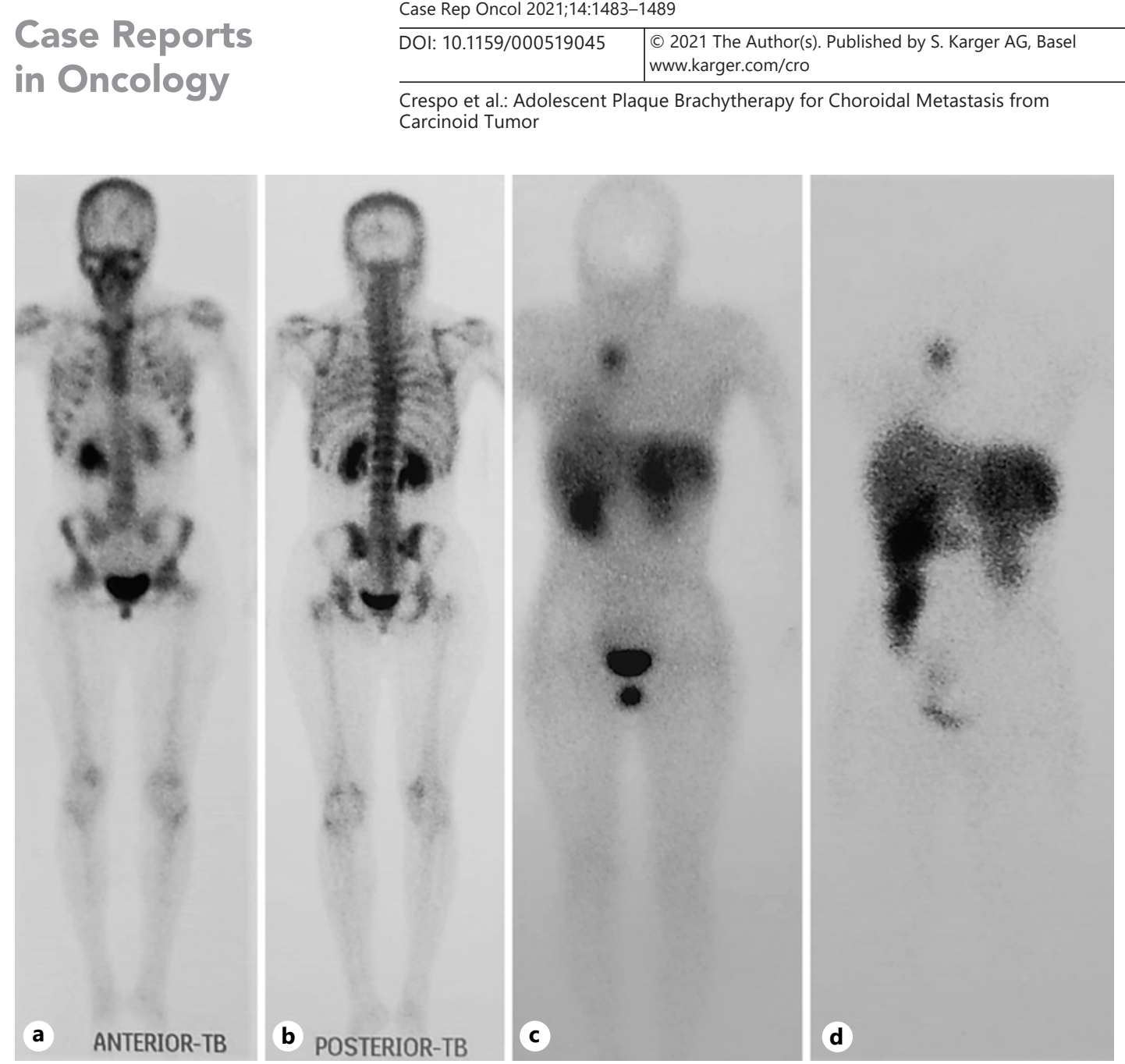

Fig. 2. a, b Bone scan showing increased uptake around both orbits, multiple ribs, L3 vertebral body, and pelvic bones. c, d Octreoscan images demonstrate areas of increased radiotracer uptake in the superior mediastinum, right lobe of the liver, and right sacrum.

Table 1. Systemic chemotherapy agents

\begin{tabular}{l} 
Treatment \\
\hline Capecitabine + temozolomide ( 4 cycles) \\
Everolimus + octreotide ( 4 cycles) \\
Carboplatin + etoposide ( 3 cycles) \\
Capecitabine + temozolomide (ongoing)
\end{tabular}

metastasis from carcinoid tumor include orange color and exudative retinal detachment [5]; these findings were present in our case. Previous treatments reported in the literature include photodynamic therapy [12], local resection [13], enucleation [14], systemic chemotherapy [15], argon laser ablation [5], external beam radiation [5], and brachytherapy [5]. Treatment may vary depending on intraocular tumor burden, location, and laterality [16]. Small tumors with shallow subretinal fluid may be amenable to laser therapy. However, larger tumors with extensive subretinal fluid may not be responsive to laser therapy.

The first report of an adolescent subject with choroidal metastasis from carcinoid tumor was described by Fu et al. [8]. The subject had bilateral multifocal disease and was treated with systemic chemotherapy (5-fluorouracil and streptozotocin) at 6-week intervals without any progression in tumor burden 5 months after diagnosis. However, long-term follow-up was not available [8]. Lack et al. [9] reported on a child with bilateral choroidal metastasis 


\section{Case Reports in Oncology}

\begin{tabular}{l|l}
\hline Case Rep Oncol 2021;14:1483-1489 \\
\hline DOI: 10.1159/000519045 & $\begin{array}{l}\text { ○ 2021 The Author(s). Published by S. Karger AG, Basel } \\
\text { www.karger.com/cro }\end{array}$ \\
\hline
\end{tabular}

Crespo et al.: Adolescent Plaque Brachytherapy for Choroidal Metastasis from Carcinoid Tumor
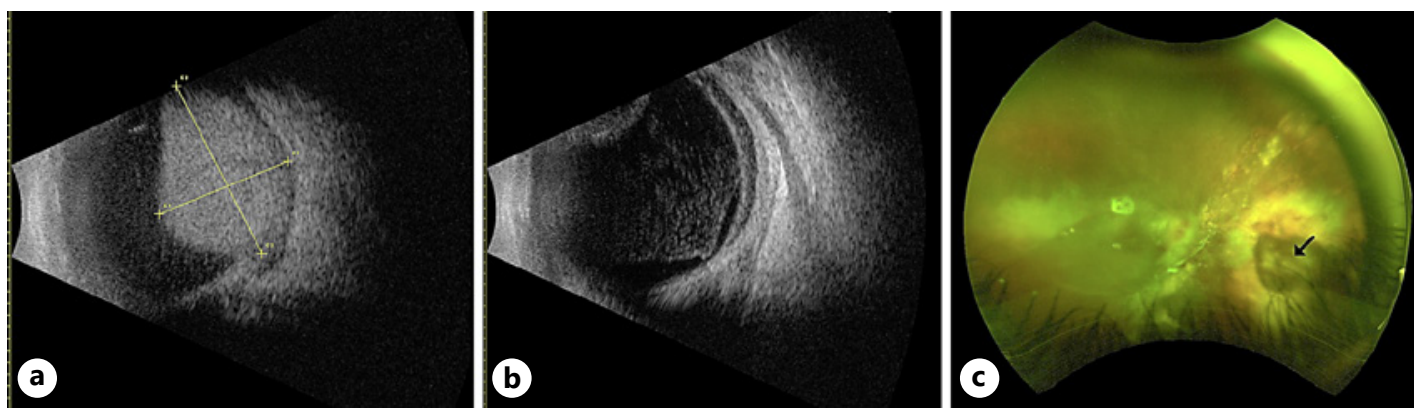

Fig. 3. a Ten months after primary therapy, B-scan shows tumoral progression with an apical height of $11.8 \mathrm{~mm}$. b One month after secondary plaque brachytherapy, B-scan shows complete tumor regression and shallow serous retinal detachment. c Fundus photograph at the last follow-up shows tumor regression (arrow).

from carcinoid tumor that progressed to bilateral blindness despite treatment with cyclophosphamide, local radiation, and laser beam therapy. Carcinoid tumors continue to represent a significant treatment challenge mainly due to the slow growth pattern and need for long-term follow-up.

Radiotherapy has been used in the treatment of carcinoid tumors in other areas of the body with positive anatomical and symptomatic outcomes. Chakravarthy et al. [17] performed a retrospective study $(n=18)$ on the symptomatic response to radiotherapy for carcinoid tumors on multiple body sites. The study showed a positive symptomatic or anatomical response to radiation in $80 \%$ of brain metastasis from carcinoid tumors with a mean dose of 33 Gy (range, 30-45 Gy); 60\% of lung carcinoid tumors responded to an mean dose of $51 \mathrm{~Gy}$ (range, 39-55.8 Gy); 87.5\% of skin metastasis from carcinoid tumor responded to a mean dose of 10 Gy (range, 8-12 Gy); and 6/7 of liver metastasis from carcinoid tumor responded to a mean dose of 21 Gy (range, 18-24 Gy) [17]. A similar retrospective study ( $n=44)$ from Schupak and Wallner [18] showed that local control and symptomatic palliation may be achieved in the majority of subjects with unresectable metastatic carcinoid tumors. The radiation response to metastasis in the spine, brain, bone, and abdomen was $92 \%, 63 \%$, $88 \%$, and $76 \%$, respectively. There is still controversy over the risk versus benefit of radiotherapy for nonocular carcinoid tumor metastasis due to slow progression and secondary radiation morbidity [19].

The literature regarding plaque brachytherapy for choroidal metastasis from carcinoid tumor is limited. Harbour et al. [5] reported 3 adult subjects with choroidal metastasis from carcinoid tumors that underwent plaque brachytherapy with positive outcomes. Plaque brachytherapy dose ranged from 50 to $74 \mathrm{~Gy}$. Two subjects underwent primary treatment with plaque brachytherapy (apical height 9.0 and $6.0 \mathrm{~mm}$ ) with complete regression. The third subject (apical height of $6.3 \mathrm{~mm}$ ) was initially treated with 30 Gy of external beam radiation, but due to incomplete regression, successful re-treatment with plaque brachytherapy was undertaken.

Due to the rarity of choroidal metastasis from carcinoid tumor, dosing of plaque brachytherapy remains unclear. A wide dose range has been described in the literature. Aziz et al. [20] reported successful treatment with plaque brachytherapy using 45 Gy to the tumor apex, with apical height decrease from $4 \mathrm{~mm}$ to $2.6 \mathrm{~mm}$. The patient was able to maintain 20/20 vision on the affected eye and did not develop any radiation-related complications after 3 months of follow-up [20]. Dosing as high as 100 Gy to the tumor apex has been reported [21]. Because carcinoid tumors have an indolent clinical course, the main limitation associated with the literature regarding radiotherapy for choroidal metastasis from carcinoid tumors is

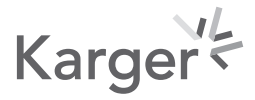


long-term follow-up. Low-dose radiation may provide short-term palliative care; however, higher doses may be needed for complete regression.

This manuscript reports the fourth subject younger than 18 years of age with choroidal metastasis from carcinoid tumor [8-10]. All cases in the literature have originated from the lungs. In contrast to previous reports, our subject had a unilateral circumscribed choroidal metastasis from a carcinoid tumor. Similarly, it is the first pediatric case to undergo plaque brachytherapy treatment. It is possible that adolescent patients with choroidal metastasis from lung carcinoid tumors may need higher radiation doses to achieve longterm regression.

\section{Conclusion}

High-dose plaque brachytherapy was efficacious for choroidal metastasis from lung carcinoid tumor in our patient and may represent a treatment option for this rare metastatic tumor. Future studies may delineate the best dosing strategy for these rare metastatic tumors.

\section{Statement of Ethics}

The study adhered to the tenets of the Declaration of Helsinki and is HIPAA compliant. Written informed consent was obtained from the patient's legal guardian for publication of this case report and any accompanying images. This case report was exempt from IRB approval.

\section{Conflict of Interest Statement}

None of the authors have a proprietary interest in this report or any potential conflicts of interest to disclose.

\section{Funding Sources}

No external funding was provided for this report.

\section{Author Contributions}

Marcos A. Crespo, MD, acquired the data and wrote and submitted the manuscript. Victor M. Villegas, MD, assisted in the writing and review of the manuscript. Maria Echevarria, MD, Carmen M. Gurrea, MD, and Timothy G. Murray, MD, assisted in the review of the manuscript. Carlos M. Chevere, MD, provided the radiation plan.

\section{Data Availability Statement}

This case report has no additional data.

\section{Karger'k}




\section{Case Reports in Oncology}

\begin{tabular}{l|l}
\hline Case Rep Oncol 2021;14:1483-1489 \\
\hline DOI: 10.1159/000519045 & $\begin{array}{l}\text { @ 2021 The Author(s). Published by S. Karger AG, Basel } \\
\text { www.karger.com/cro }\end{array}$ \\
\hline
\end{tabular}

Crespo et al.: Adolescent Plaque Brachytherapy for Choroidal Metastasis from Carcinoid Tumor

\section{References}

1 Stanford WR, Davis JE, Gunter JU, Hobart SG Jr. Bronchial adenoma (carcinoid type) with solitary metastasis and associated functioning carcinoid syndrome. South Med J. 1958 Apr;51(4):449-54.

2 Maggard MA, O'Connell JB, Ko CY. Updated population-based review of carcinoid tumors. Ann Surg. 2004 Jul; 240(1):117-22.

3 Abdel-Rahman 0. Modified staging system for pulmonary carcinoids on the basis of lung cancer TNM system. Clin Transl Oncol. 2018 May;20(5):670-7.

4 Modlin IM, Sandor A. An analysis of 8305 cases of carcinoid tumors. Cancer. 1997 Sep;79(4):813-29.

5 Harbour JW, De Potter P, Shields CL, Shields JA. Uveal metastasis from carcinoid tumor. Clinical observations in nine cases. Ophthalmology. 1994 Jun;101(6):1084-90.

6 Rojas Y, Shi YX, Zhang W, Beierle EA, Doski JJ, Goldfarb M, et al. Primary malignant pulmonary tumors in children: a review of the national cancer data base. J Pediatr Surg. 2015 Jun;50(6):1004-8.

7 Navalkele P, Odorisio MS, Odorisio TM, Zamba GK, Lynch CF. Incidence, survival, and prevalence of neuroendocrine tumors versus neuroblastoma in children and young adults: nine standard SEER registries, 19752006. Pediatr. Blood Cancer. 2010 Jan;56(1):50-7.

8 Fu YS, McWilliams NB, Stratford TP, Kay S. Bronchial carcinoid with choroidal metastasis in an adolescent. Case report and ultrastructural study. Cancer. 1974 Mar;33(3):707-15.

9 Lack EE, Harris GB, Eraklis AJ, Vawter GF. Primary bronchial tumors in childhood. A clinicopathologic study of six cases. Cancer. 1983 Feb;51(3):492-7.

10 Kumar A, Pal N, Sharma YR, Gupta R, Jain V, Sharma R. Bronchial carcinoid tumour with choroidal metastasis masquerading as ocular tuberculosis. Eye. 2006 Sep;20(9):1101-3.

11 Bloch RS, Gartner S. The incidence of ocular metastatic carcinoma. Arch Ophthalmol. 1971 Jun;85(6):673-5.

12 Harbour JW. Photodynamic therapy for choroidal metastasis from carcinoid tumor. Am J Ophthalmol. 2004 Jun;137(6):1143-5.

13 Bardenstein DS, Char DH, Jones C, Crawford JB, Miller TR, Riekhof FT. Metastatic ciliary body carcinoid tumor. Arch Ophthalmol. 1990 Nov;108(11):1590-4.

14 Riddle PJ, Font RL, Zimmerman LE. Carcinoid tumors of the eye and orbit: a clinicopathologic study of 15 cases, with histochemical and electron microscopic observations. Hum Pathol. 1982 May;13(5):459-69.

15 Fan JT, Ortiz RG, Buettner H. Regression of choroidal metastases from a bronchial carcinoid tumor after chemotherapy with cisplatin and etoposide. Am J Ophthalmol. 1994 Jan;117(1):111-3.

16 Arepalli S, Kaliki S, Shields CL. Choroidal metastases: origin, features, and therapy. Indian J Ophthalmol. 2015 Feb;63(2):122-7.

17 Chakravarthy A, Abrams RA. Radiation therapy in the management of patients with malignant carcinoid tumors. Cancer. 1995 Mar;75(6):1386-90.

18 Schupak KD, Wallner KE. The role of radiation therapy in the treatment of locally unresectable or metastatic carcinoid tumors. Int J Radiat Oncol Biol Phys. 1991 Mar;20(3):489-95.

19 Martini N, Zaman MB, Bains MS, Burt ME, Mccormack PM, Rusch VW, et al. Treatment and prognosis in bronchial carcinoids involving regional lymph nodes. J Thorac Cardiovasc Surg. 1994 Jan;107(1):1-7.

20 Aziz HA, Martel JN, Biscotti CV, Singh AD, Shields CL. This, that, or something different? Surv Ophthalmol. 2015 Sep;60(5):495-9.

21 Fan JT, Buettner H, Bartley GB, Bolling JP. Clinical features and treatment of seven patients with carcinoid tumor metastatic to the eye and orbit. Am J Ophthalmol. 1995 Feb;119(2):211-8. 